\title{
Spectroscopic study on Thiourea and Thiosemicarbazide in Non- aqueous media
}

\author{
Ragamathunnisa . $\mathrm{M}_{2}^{1}$ Jasmine Vasantha Rani.E ${ }^{2}$, Padmavathy $\mathrm{R}^{2}$, Radha $\mathrm{N}^{3}$ \\ ${ }^{1}$ (Government Arts College for Women, Department of Physics, Pudukkottai, Tamilnadu, India) \\ ${ }^{2}$ (Seethalakshmi Ramaswamy College, Department of Physics, Trichy Tamilnadu, India) \\ ${ }^{3}$ (Seethalakshmi Ramaswamy College, Department of chemistry, Trichy Tamilnadu, India)
}

\begin{abstract}
The spectroscopic methods of the different regions of the Electromagnetic spectrum are the most powerful and useful techniques available for the understanding of molecular structure, nature of bonding between atoms, confirmation analysis, symmetry of molecules, ions in crystals and so forth. The high resolution $I R$ spectroscopic studies have recently received a great thrust due to the advent of advanced Fourier transform spectrometers. In the present investigation the spectral changes are studied in the vibrational spectra of thiourea, thiosemi carbazide in non aqueous solvent. The $\mathrm{H}_{2} \mathrm{~N}$ stretching vibrations observed at $3371 \mathrm{~cm}^{-1}$, $3259 \mathrm{~cm}^{-1}, \& 3156 \mathrm{~cm}^{-1}$ indicated that 3 different types of $\mathrm{NH}_{2}$ groups arising out of tautomeric form. The results obtained from the present study confirm that the solute - solvent interactions occurring in the solution is through intermolecular $H$ - bonding.
\end{abstract}

Keywords - Dimeric structure, Hydrogen bonding, Thiosemicarbazide, Thiourea.

\section{Introduction}

Progress in the area of non-linear optics (NLO) depends upon the development of new materials. When compared with the inorganic materials, organic and materials are attracting a great deal of attention, as they have large optical susceptibilities, inherent ultra fast response time and good optical properties [1-3]. The advent of the instrumental methods like infrared has now made the task of an organic chemist comparatively simpler [4] although the IR spectrum is characteristic of the entire molecule. Fourier transform Infrared Spectrometry (FTIR) has been extensively developed over the past decade and provides a number of advantages. The Organo sulfur compounds have variety of applications in industries. It is mainly used in textile processing [5] and also in the reductive work up of Ozonolysis [6] to give carbonyl compound. They are also used as building blocks to pyrimidine derivatives. Thiourea condenses with $\beta$-dicarbonyl compounds [7]. Thiourea is a reagent in organic synthesis to pharmaceuticals like thiobarbituric acid and sulfathiazole. Thiosemicarbozide has been shown to be good ligand for range of metals, including zinc, mercury, cadmium and nickel $[8,9]$ Thiosemicarbazide is a thiourea derivative. It is used in the preparation of antibacterial compounds. In the present investigation the FTIR spectral studies are carried out in non - aqueous solutions of thiourea(Thiourea solvated in formamide) \& thiosemicarbazide (Thiosemicarbazide in formamide) in order to probe the effect of $\mathrm{H}$ - bonding in the solvation process.

\section{Experimental Technique}

Thiourea \& Thio semicarbazide (AR Merc) were used in non - aqueous medium. Solutions of saturation molalities were prepared by weighing in electronic balance and an accuracy of $(0.001 \mathrm{gm})$ were stored in vaccum decicator until use. The Fourier transform infrared was carried out between $4000 \mathrm{~cm}^{-1}$ to $400 \mathrm{~cm}^{-1}$ by recording the spectrum using PERKIN ELMER SPECTRUM RXI spectrometer.

\subsection{FTIR Study - THIOUREA (TU)}

\section{Results And Discussion}

The spectrum of formamide (solvent) and thiourea(solute) is shown in fig. (1) and (2).The observed frequencies are tabulated. In the spectrum of thiourea, the vibrations found at $3371 \mathrm{~cm}^{-1}, 3260 \mathrm{~cm}^{-1}, \&$ $3156 \mathrm{~cm}^{-1}$ indicate that the three different types of $\mathrm{NH}_{2}$ groups arising out of tautomeric form. The peaks at $2684 \mathrm{~cm}^{-1}, 2354 \mathrm{~cm}^{-1}$ besides small shoulders upto $2100 \mathrm{~cm}^{-1}$ are indicative of ammonium \& iminium salt like structure. A sharp peak at $1585 \mathrm{~cm}^{-1}$ arises due to thioamide $(\mathrm{C}=\mathrm{S})$ asymmetric stretching vibration while the symmetric vibrations may be located at $1449 \mathrm{~cm}^{-1}$. A broad band at $1088 \mathrm{~cm}^{-1}$ is due to C-S vibrations. The sharp peak at $729 \mathrm{~cm}^{-1}$ is assigned to the S-H bending vibration. C-N bending vibration and NH bending vibration found at $627 \mathrm{~cm}^{-1}$ and at $493 \mathrm{~cm}^{-1}$ respectively. 


\subsection{FTIR- THE SOLUTION OF THIOUREA IN FORMAMIDE:}

The solution spectrum is shown in fig. (3) In the solution the solvent peak at $3416 \mathrm{~cm}^{-1}$ has shifted to $3372 \mathrm{~cm}^{-1}$ towards lower energy side by $44 \mathrm{~cm}^{-1}$. Besides a series of stretching vibrations are observed at $3267 \mathrm{~cm}^{-1}, 3163 \mathrm{~cm}^{-1}, 2888 \mathrm{~cm}^{-1}, 2688 \mathrm{~cm}^{-1}$ and at $2204 \mathrm{~cm}^{-1}$. These vibration frequencies are confirmed the presence of both the solute $[\mathrm{NH}]$ and solvent peaks $[\mathrm{CH}]$ and $[\mathrm{C}=\mathrm{NH}]$. The sharp peak of bands at $1697 \mathrm{~cm} \neg^{1}$ and $1550 \mathrm{~cm} \neg^{1}$ in the solvent spectrum have disappeared and a new band occurs at $1598 \mathrm{~cm} \neg^{1}$. This peak has arisen due to $\mathrm{H}$ - bonding between $>\mathrm{C}=\mathrm{O}$ of solvent and $\mathrm{NH}_{2}$ of solute destroying the dimeric structure of pure solvent. The $>\mathrm{C}=\mathrm{S}$ vibration at $1584 \mathrm{~cm} \neg^{1}$ is shifted to higher energy by $14 \mathrm{~cm} \neg^{1}$. A higher energy shift of $10 \mathrm{~cm} \neg^{1}$ occurs at $1399 \mathrm{~cm} \neg^{1}$ peak and dramatic change in this band region may be attributed to solute- solvent interaction in $>\mathrm{C}=\mathrm{N}-\mathrm{H}$ site. A new peak arises at $1077 \mathrm{~cm} \neg^{1}$ due to C-S stretching mode of the solute. The 729 $\mathrm{cm} \neg^{1} 627 \mathrm{~cm} \neg^{1}$ are due to bending vibrations of the solute shifted to lower energy region of the solution compared to the salt spectrum. In this region the $494 \mathrm{~cm} \neg^{1}$ has shifted to higher energy region by $1 \mathrm{~cm} \neg^{1}$. All these spectral changes may be due to the H-bonding pattern which shown below<smiles></smiles><smiles></smiles>

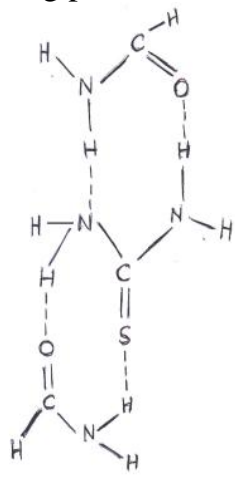<smiles>NP1CNC(=S)NNCN1</smiles>

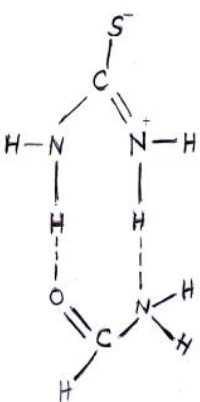

\subsection{FTIR - THIOSEMI CARBAZIDE (TSC)}

The salt spectrum of TSC is shown in fig. (4) The series of vibrations in the region $3365 \mathrm{~cm}^{-1}$ to 3169 $\mathrm{cm}^{-1}$ are due to different types of $\mathrm{NH}_{2}$ and $\mathrm{NH}$ groups. The absorption frequencies found at $1616 \mathrm{~cm}^{-1}$ and 1516 $\mathrm{cm}^{-1}$ are due to $>\mathrm{C}=\mathrm{S}-\mathrm{NH}$ functional groups. ie. asymmetric and symmetric stretching vibrations of thio amide I \& II bands. At $1273 \mathrm{~cm}^{-1} \& 1155 \mathrm{~cm}^{-1} \mathrm{C}=\mathrm{N}$ bending and C-S stretching vibrations occur. The $\mathrm{C}-\mathrm{S}, \mathrm{NH}_{2}$ and $\mathrm{NH}$ bending vibrations are found at $993 \mathrm{~cm}^{-1}, 792 \mathrm{~cm}^{-1}, 649 \mathrm{~cm}^{-1}$ and $576 \mathrm{~cm}^{-1}$ respectively.

\subsection{FTIR - THIOSEMICARBAZIDE IN FORMAMIDE (TSC)}

The solution spectrum of TSC in formamide is shown in fig. (5), which is similar to the solvent rather than to the salt. This indicates the predominant amount of formamide compared to solute molecules. A lower energy shift of $3400 \mathrm{~cm} \neg^{1}$ band by $16 \mathrm{~cm} \neg^{1}$ is observed. It is due to the weakening of $\mathrm{C}=\mathrm{O}-\mathrm{NH}$ bonds in the solvent. Broadening of this peak which is quite smooth may be due to the binding of $\mathrm{NH}$ of formamide to another group in the solute. The four small shoulders like peaks in the region $2407 \mathrm{~cm} \neg^{1}$ to $2064 \mathrm{~cm} \neg^{1}$ have become well defined in the solution spectra indicating the presence of strong solute - solvent interactions. A broad peak centered at $1619 \mathrm{~cm} \neg^{1}$ encompasses both the amide I of formamide and thioamide I band of TSC. The intense peak is characteristics of strong solute - solvent interaction at $>\mathrm{C}=\mathrm{O} \&>\mathrm{C}=\mathrm{S}$ groups. This peak has shifted to lower energy region by $79 \mathrm{~cm} \neg^{1}$ indicating $>\mathrm{C}-\mathrm{O}$ more single bond character during donar - acceptor interaction. Similarly the amide II vibration has also shifted to lower energy by $22 \mathrm{~cm} \neg^{1}$ indicating lengthening of bonds. At $997 \mathrm{~cm} \neg^{1}, 787 \mathrm{~cm} \neg^{1}$, the solute vibrations are observed. The former is higher energy shift by 3.6 $\mathrm{cm} \neg^{1}$, while the later peak is lower energy shift by $4.5 \mathrm{~cm} \neg^{1}$ which is the indication of strong binding. The solute - solvent interaction through inter molecular $\mathrm{H}$ bonding are given below. 

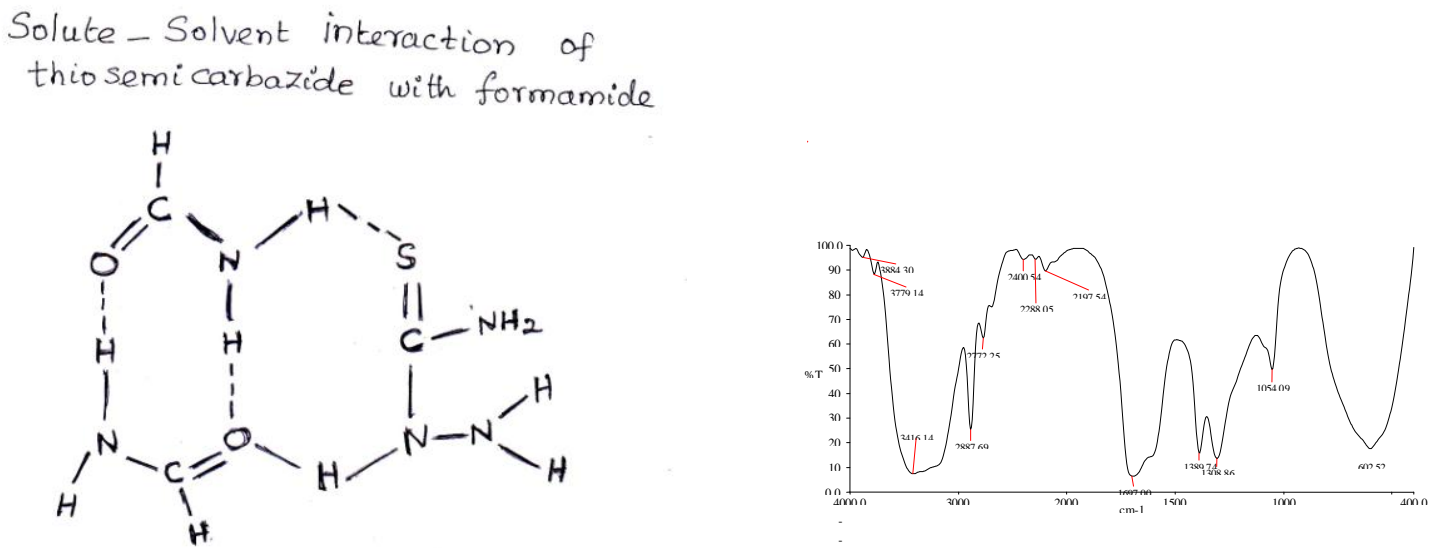

Fig.1 FTIR Spectrum of Formamide

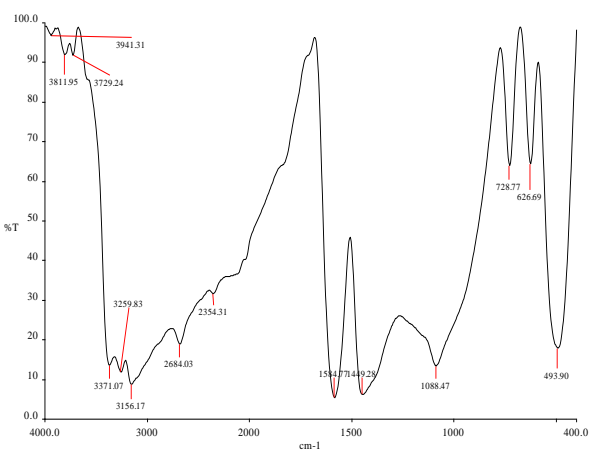

Fig.2 FTIR Spectrum of Thiourea

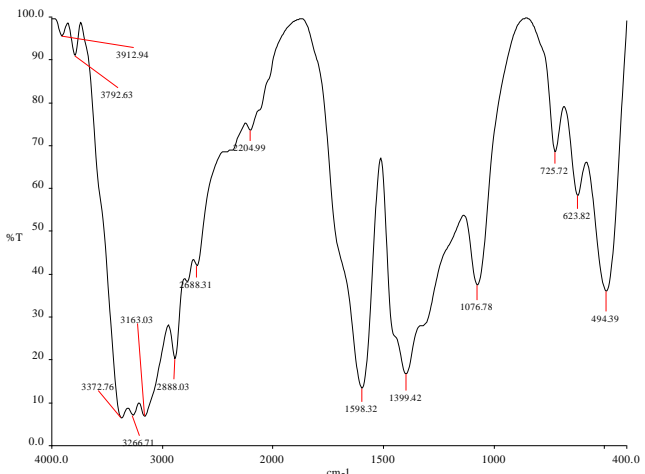

Fig.3 FTIR Spectrum of Thiourea in Formamide

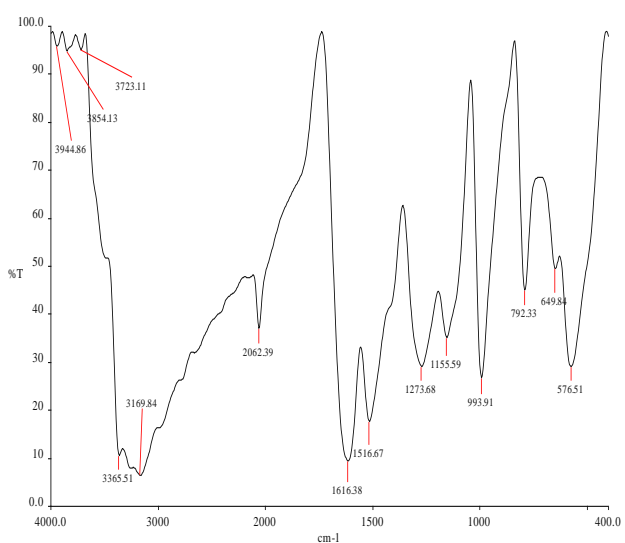

Fig.4 FTIR Spectrum of Thiosemicarbazide

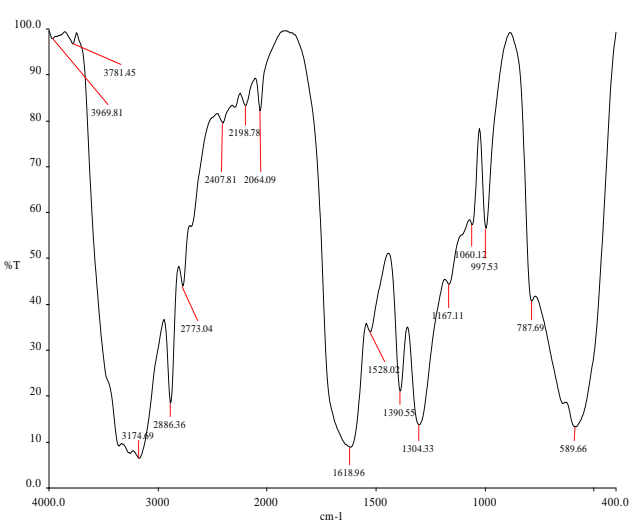

Fig.5 FTIR Spectrum of Thiosemicarbazide in Formamide 


\section{TABLE - 1 A comparative study of FTIR spectral results}

Wave Number $\mathrm{cm}^{-1}$

\begin{tabular}{|l|l|l|l|l|l|}
\hline $\begin{array}{l}\text { Band } \\
\text { Assignment }\end{array}$ & Formamide & Thio urea & $\begin{array}{l}\text { Thio urea with } \\
\text { Formamide }\end{array}$ & TSC & $\begin{array}{l}\text { TSC with } \\
\text { Formamide }\end{array}$ \\
\hline$v_{\mathrm{NH}}$ & 3416 & 3371 & 3372 & 3366 & $3400(\mathrm{sh})$ \\
\hline$v_{\mathrm{CH}}$ & 2888 & 3260 & 3267 & 3169 & 2886 \\
& 2772 & 3156 & 3163 & $2400-$ & 2773 \\
& $2600-2500(\mathrm{sh})$ & 2684 & 2888 & $2300(\mathrm{sh})$ & $2600(\mathrm{sh})$ \\
& 2400 & 2354 & 2688 & $2062(\mathrm{sh})$ & 2408 \\
\hline$v_{\mathrm{C}=\mathrm{N}}$ & 2288 & 2100 & 2205 & 1616 & $2300-2200(\mathrm{sh})$ \\
& 2197 & $1700-$ & 1598 & 1517 & 1618 \\
\hline$v_{\mathrm{C}=\mathrm{S}}$ & 1697 & $1600(\mathrm{sh})$ & 1377 & $1356(\mathrm{sh})$ & 1528 \\
& & & & & \\
\hline$v_{\mathrm{C}-\mathrm{S}}$ & $1550(\mathrm{sh})$ & 1584 & $1400-$ & 1273 & 1391 \\
& 1390,1309 & 1449 & $1300(\mathrm{sh})$ & 993 & 1304 \\
& 1054 & 1088 & 726 & 792 & 1060 \\
\hline$v_{\mathrm{CH}}$ & 603 & 729 & 624 & 650 & 997 \\
& & 627 & 494 & 577 & 787 \\
& & 494 & & & 590 \\
\hline
\end{tabular}

\section{Conclusion}

From this spectral analysis various functional groups are identified. The dimeric structure of formamide is changed due to solvation with thiourea. Strong solute-solvent interaction occurs in TSC with formamide. This study also confirmed that the solute- solvent interactions occurring in the solutions is through intermolecular H-bonding.

\section{Acknowledgement}

Authors acknowledge the immense help received from the scholars whose articles are citied and included in references of this manuscript. The authors also grateful to authors/editors/publishers of all those articles, journals and books from where the literature for this article has been reviewed and discussed.

\section{References}

[1] S.Dhanuskodi, PAA. Mary, J.Cryst Growth (253); 2003 (424).

[2] PN.Prasad, DJ.Williams, Introduction to Nonliear Optical Effects in Molecules and Polymers, john- Wiley\& sons inc., New York, (35-38) 1991.

[3] N.Vijayan, R.Ramesh Babu, M.Gunasekaran, R.Gobalakrishnan, P.Ramaswamy, J.Cryst.Growth 256: 2003 (174-182)

[4] M.Silverstein, X.Webster "Spectroscopic identification of organic compound" $6^{\text {th }}$ Edition John Wiley\& Sons, Inc, New York (1998)

[5] B. Mertschenk, F.Beck, W.Bauer'Thiourea and Thiourea Derivatives" in Ullmann's Encyclopedia of Industrial chemistry by Wiley- VCH Verlag GmbH \&Co.KGaA.Allrights reserved. DOI: 10.1002/14356007.a26-803 (2002)

[6] D.Gupta, G.Soman, and S.Dev. (1982)."Thiourea, a convenient reagent for the reductive cleavage Of olefin ozonolysis products". Tetrahedron 38:3013.Doi:10.1016/0040-4020 (82)80187-7 (1982)

[7] H.M.Foster, and H.R.Snyder, Coll.Vol. 4: 1963(638)

[8] DS.Mahadevappa, S.Ananda Murhy, J.Austr, Chem.:25: 1972(1565-1568)

[9] R.E.Ballard, DB.Powell, UA.Jeyasooriya, Acta Cryst: B30:1974 (1111-1113) 